\title{
The LHCb RICH system: current detector performance and status of the upgrade program
}

\author{
Massimiliano Fiorini* ${ }^{*}$ \\ Università degli Studi di Ferrara and INFN Sezione di Ferrara \\ E-mail: Massimiliano.Fiorini@cern.ch
}

\begin{abstract}
$\mathrm{LHCb}$ is a precision experiment devoted to the study of $\mathrm{CP}$ violation and rare decays of $\mathrm{b}$ and $\mathrm{c}$ quarks, and to the search for new physics beyond the Standard Model at the Large Hadron Collider (LHC) at CERN. The Ring-Imaging Cherenkov (RICH) system is a key component of the LHCb experiment: it consists of two RICH detectors that provide charged particle identification over a wide momentum range (2-100 GeV/c) and angular acceptance (15-300 mrad). The LHCb RICH system has been performing extremely well during Run 1 and is providing the LHCb experiment also in Run 2 with a robust, reliable and precise particle identification system. Performance of the $\mathrm{RICH}$ detectors measured from data will be presented, with special reference to its dependence on calibration parameters and event multiplicities. The $\mathrm{LHCb}$ experiment is preparing for an upgrade during the second LHC long shutdown (2019-2020) in order to fully exploit the LHC flavour physics potential. A five-fold increase in instantaneous luminosity is foreseen reaching $2 \times 10^{33} \mathrm{~cm}^{-2} \mathrm{~s}^{-1}$. In order to achieve the planned $40 \mathrm{MHz}$ continuous data taking, a substantial change in the $\mathrm{LHCb}$ trigger and read-out schemes will be implemented. The RICH detectors will require new photo-detectors and electronics. The optics will also be slightly modified for improved performance at the higher luminosity. Tests of the complete photo-electronic chain have been performed in dedicated test beams in 2014-16. Status and perspectives of the RICH upgrade program will be presented.
\end{abstract}

$38^{\text {th }}$ International Conference on High Energy Physics

3-10 August 2016

Chicago, USA

\footnotetext{
* Speaker.

${ }^{\dagger}$ On behalf of the LHCb RICH Collaboration
} 


\section{Introduction}

The LHCb experiment is designed to study CP violation and rare decays of heavy flavours at the CERN Large Hadron Collider [1]. The LHCb detector acceptance spans the polar angles between $15 \mathrm{mrad}$ to $300 \mathrm{mrad}$ in the horizontal bending plane of the spectrometer magnet, and $250 \mathrm{mrad}$ in the vertical non-bending plane, equivalent to a pseudorapidity range $2<\eta<5$. Although the acceptance corresponds to only $4 \%$ of the solid angle, it covers about $40 \%$ of the $b \bar{b}$ production.

The Ring Imaging Cherenkov (RICH) detector [2] has the primary role of identifying charged particles in a wide momentum range $(2-100 \mathrm{GeV} / \mathrm{c})$ and is composed of two separate systems: RICH 1, located close to the interaction point and covering the full acceptance of the experiment and the 2-50 GeV/c momentum region, and $\mathrm{RICH} 2$, placed after the spectrometer magnet and covering up to $120 \mathrm{mrad}$ angular acceptance and the $15-100 \mathrm{GeV} / \mathrm{c}$ momentum range.

\section{RICH detector operation and performance}

LHCb operated at tunable levelled luminosities up to $4 \times 10^{32} \mathrm{~cm}^{-2} \mathrm{~s}^{-1}$ during Run1 (20102012), a factor of two higher than the design value, and accumulated an integrated luminosity of about $3 \mathrm{fb}^{-1}$. During Run 2 (2015-2018) LHCb is expected to collect another $5 \mathrm{fb}^{-1}$.

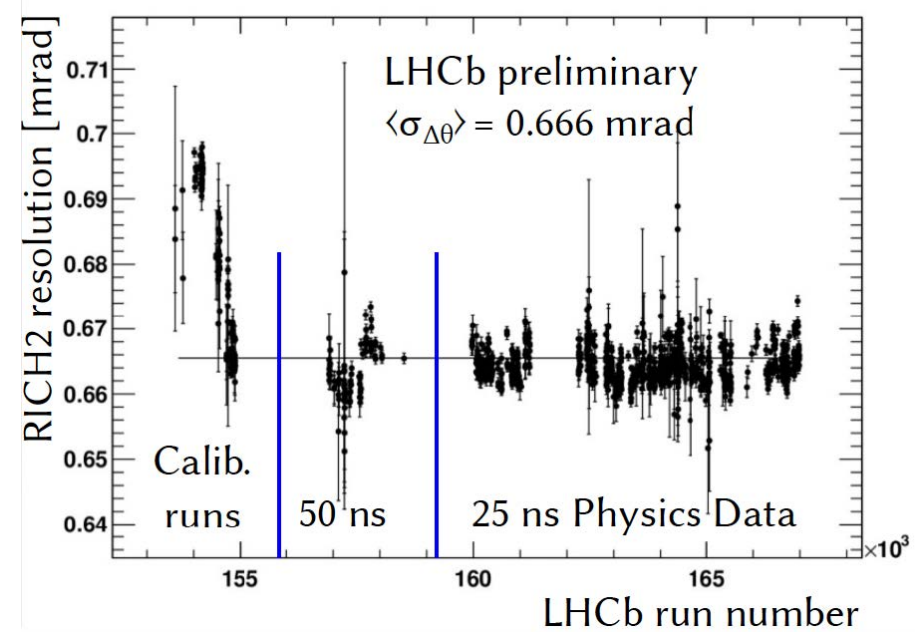

Figure 1: Time dependence of the Cherenkov angle resolution for RICH 2 during part of the 2015 data taking.

The RICH detector operates in a high multiplicity environment, with an average number of photon hits per event of about 2000 in each of the two detectors. Calibrations are necessary to achieve the optimal detector performance. Since the beginning of Run2 calibrations are performed online on a run by run basis (a run is typically limited to 1 hour) and are completely automated: an online reconstruction is performed in real time, calibrations are computed from this and then provided to the high level trigger system to complete the event processing.

The refractive index of the radiators may change due to temperature, pressure and gas mixture variations. The distribution of the difference between the measured and expected Cherenkov angles 
for saturated $(\beta=1)$ tracks is fitted with a gaussian curve for signal superimposed to a polynomial background. The mean is extracted from the fit and any deviation from zero is assumed to be a mismodeling of the gas parameters, so a refractive index scale factor is computed to bring the distribution back to zero. An example of the time variation of the Cherenkov angle resolution for RICH 2 is shown in Fig. 1 for different 2015 data taking periods.

The position of the Hybrid Photon Detectors (HDPs [3]) photocathode image on the anode can change due to charging effects. A large number of events are integrated to produce a continuous image of the photocathode. Due to occupancy variations in space the images are not uniformly illuminated so the procedure has to be robust in finding the edges: images are cleaned and a Sobel filter is used to detect the edge, then the parameters of the photocathode image are determined from a fit and automatically updated.

In addition the HPDs are subject to image distortions due to fringe magnetic fields of the experiment dipole magnet. A light bar moves across the HPD array projecting test patterns using a grid of light-emitting diodes. The correction is parameterised by a polynomial in the radial and angular coordinates, the parameters of which are determined from a fit to the measured points such that they reproduce the grid pattern. The procedure is not automated, and is performed only once a year for each magnetic field direction.

Mirrors alignment is an important ingredient to get the best possible resolution in reconstructing Cherenkov angles. For a correctly aligned system the Cherenkov angle should not depend on the angle around the photons ring, but in the case of misalignments the Cherenkov angle varies periodically depending on that angle. These variations are fitted to produce first order corrections to the alignments, and the procedure is iterated until further changes are below a threshold. The mirrors alignment procedure is running automatically via software on fill by fill basis. However corrections are currently not automatically applied to the data, but just used to monitor how the alignment is changing throughout the year.

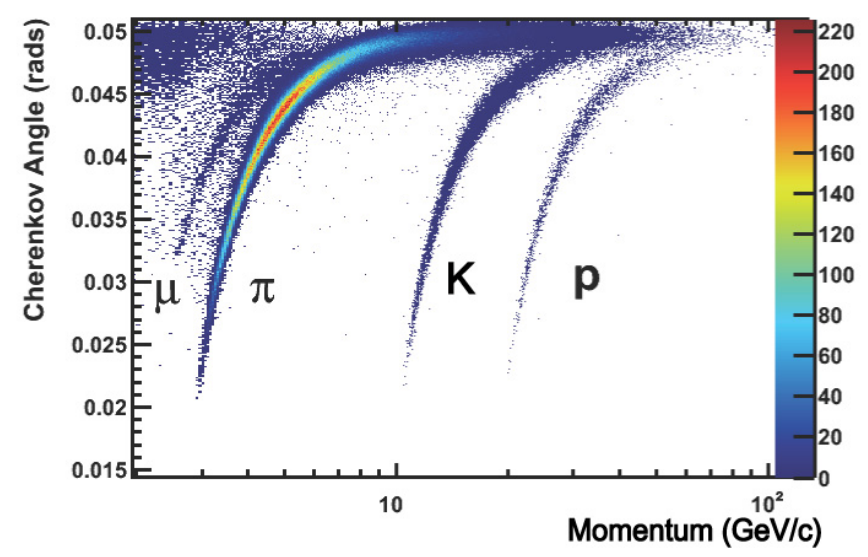

Figure 2: Distribution of Cherenkov angles as a function of the track momentum for isolated tracks in RICH 1. Different charged particles species are clearly identified.

The detector showed excellent performance during Run1 with a small improvement in particle identification in Run2. A single photon angular resolution of about $1.6 \mathrm{mrad}$ is measured for the gas radiator in $\mathrm{RICH} 1\left(\mathrm{C}_{4} \mathrm{~F}_{10}\right)$ and about $0.7 \mathrm{mrad}$ for $\mathrm{RICH} 2\left(\mathrm{CF}_{4}\right)$. A distribution of the Cherenkov 
angles as a function of particle momenta is shown in Fig. 2 for isolated tracks in RICH 1: different charged particles are clearly identified and separated in distinct bands.

\section{Upgrade of the RICH detector}

The LHCb detector will undergo a major upgrade in 2019-20 to allow operations at a luminosity of $2 \times 10^{33} \mathrm{~cm}^{-2} \mathrm{~s}^{-1}$ [4]. The detector upgrade will result in a substantial change of the read-out and trigger architecture which will allow reading out the full detector at $40 \mathrm{MHz}$.

In order to adapt the RICH for running at higher luminosity, the focal length of the spherical mirrors for RICH 1 will be increased to reduce hit occupancy. The optical system and the support mechanics and cooling will be redesigned [5]. The HPDs will be replaced by commercially available Multi-anode Photo-Multiplier Tubes (Ma-PMTs) using new front-end electronics based on the CLARO8 chip [6], an FPGA-based digital board and the GBT chip [7] for data transmission.

The upgraded RICH detector will use two kinds of Ma-PMTs produced by Hamamatsu Photonics: the R11265 (1", 64 pixels) for the whole RICH 1 and the inner region of RICH 2, and the R12699 (2", 64 pixels) for the outer - and lower occupancy - region of RICH 2. The typical gain of these tubes is about $1 \times 10^{6}$ at $1 \mathrm{kV}$, and a gain spread of $1: 3$ has been measured between pixels in the same PMT, in addition to a 1:3 gain spread between different PMTs.

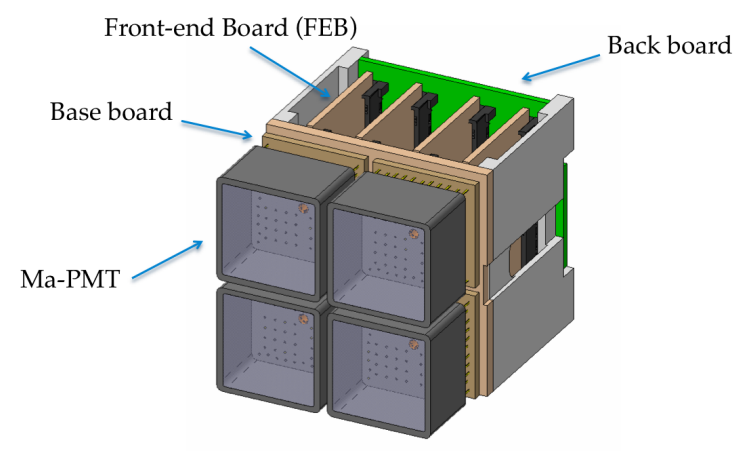

Figure 3: Drawing of one elementary cell for 1" Ma-PMTs.

A front-end electronics system capable of single photon counting at $40 \mathrm{MHz}$ with the above Ma-PMTs is needed, able to compensate for the spread in gain between pixels and the loss of performance from the accumulated radiation dose expected during the upgrade phase (200 krad, $3 \times 10^{12} 1 \mathrm{MeV} \mathrm{n}_{e q} / \mathrm{cm}^{2}$ and $1.2 \times 10^{12} \mathrm{HEH} / \mathrm{cm}^{2}$, corresponding to $50 \mathrm{fb}^{-1}$ integrated luminosity). The CLARO8 chip is an application specific integrated circuit (ASIC) designed for single-photon counting up to $40 \mathrm{MHz}$, with a peaking time of $5 \mathrm{~ns}$, a recovery time better than $25 \mathrm{~ns}$ and a power consumption of about $1 \mathrm{~mW}$ per channel. The CLARO 8 has 8 channels and a configuration register protected against single event upsets by triple modular redundancy and has been extensively tested for radiation hardness [8].

The basic photo-detector element is represented by the so-called Elementary Cell (EC), with two versions for the small and large Ma-PMTs. Fig. 3 shows a schematic drawing of the EC for the 1" tubes, which comprises 4 Ma-PMTs, 1 base-board, 1 back-board, 4 front-end boards housing the CLARO8 chips, and the mechanical structure. A magnetic shield, not shown in the picture, completes the EC. 


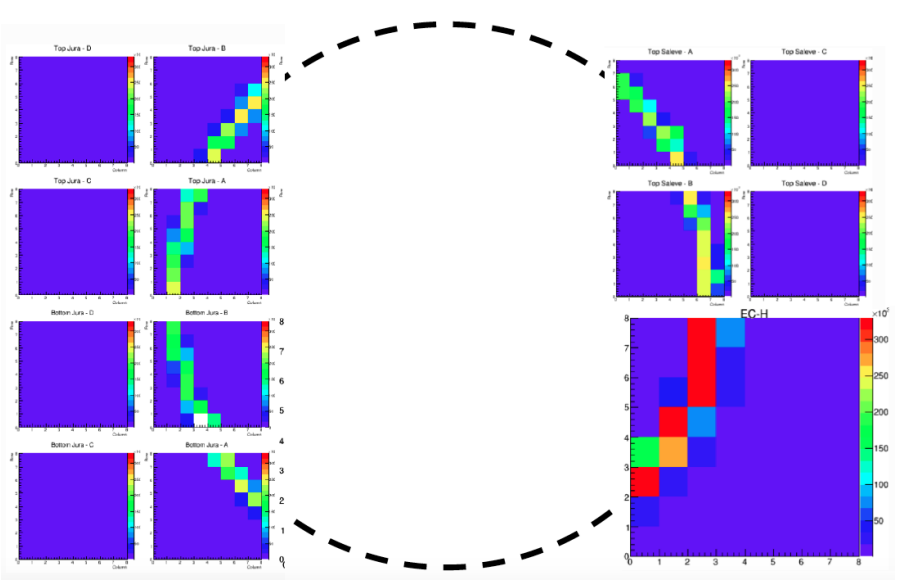

Figure 4: Cumulative distribution of Cherenkov photons over four EC during the 2016 testbeam, integrated over a large number of events.

A compact prototype RICH detector was built and tested in 2014-2016 to measure the performance of the close-to-final opto-electronics chain in a test beam. The system was made of a solid radiator and few Elementary Cells of both kinds, and allowed the operation of a prototype detector in a realistic environment. The cumulative hit distribution for the configuration used in 2016 is shown in Fig. 4. Extensive tests were performed on calibration procedures, noise and thermal measurements, and Cherenkov ring fitting, allowing the validation of the design of both EC types in view of the production phase.

\section{Conclusions}

The LHCb RICH detectors have been operating with high efficiency in a high multiplicity environment since the start of LHC operation and form an essential part of the experiment. Since the beginning of Run2, calibrations and alignments are calculated and applied online. The LHCb RICH upgrade program, designed to operate the detector in an environment with a luminosity of $2 \times 10^{33} \mathrm{~cm}^{-2} \mathrm{~s}^{-1}$, is progressing well and is on schedule for installation during the 2019-20 LHC shutdown. New photo-detectors and a new electronics chain will be used for reading out the full RICH detector at $40 \mathrm{MHz}$. Modifications to the optical and mechanical systems are also underway. Very successful tests with charged particle beams allowed to validate the close-to-final opto-electronics chain in realistic conditions.

\section{References}

[1] A. A. Alves Jr. et al. [LHCb Collaboration], "The LHCb Detector at the LHC”, JINST 3 (2008) S08005

[2] M. Adinolfi et al. [LHCb RICH Group Collaboration], "Performance of the LHCb RICH detector at the LHC', Eur. Phys. J. C 73 (2013) 2431

[3] S. Eisenhardt, "Production and tests of Hybrid Photon Detectors for the LHCb RICH detectors", Nucl. Instrum. Meth. A 595 (2008) 142 
[4] The LHCb Collaboration (2011), "Letter of Intent for the LHCb Upgrade", CERN-LHCC-2011-001, LHCC-I-018

[5] The LHCb Collaboration (2013), "LHCb PID Upgrade Technical Design Report", CERN-LHCC-2013-022, LHCB-TDR-014

[6] P. Carniti et al., "CLARO-CMOS, a very low power ASIC for fast photon counting with pixellated photodetectors", JINST 7 (2012) P11026

[7] P. Moreira, A. Marchioro, K. Kloukinas, "The GBT, a proposed architecture for multi-Gbps data transmission in high energy physics", Proceedings of the TWEPP-07 Topical Workshop on Electronics for Particle Physics

[8] M. Andreotti et al., "Irradiation of the CLARO-CMOS chip, a fast ASIC for single-photon counting", Nucl. Instrum. Meth. A 787 (2015) 234 